\title{
Real Time, Non-Invasive Assessment of Leaflet Deformation in Heart Valve Tissue Engineering
}

\author{
J. Kortsmit, ${ }^{1}$ N. J. B. Driessen, ${ }^{1}$ M. C. M. Rutten, ${ }^{2}$ and F. P. T. BaAijens ${ }^{3}$ \\ ${ }^{1}$ Laboratory for Biomechanics and Tissue Engineering, Department of Biomedical Engineering, Eindhoven University of \\ Technology, P.O. Box 513, Building W-hoog 4.105, 5600 MB Eindhoven, The Netherlands; ${ }^{2}$ Laboratory for Biomechanics and \\ Tissue Engineering, Department of Biomedical Engineering, Eindhoven University of Technology, P.O. Box 513, Building \\ W-hoog 4.120, 5600 MB Eindhoven, The Netherlands; and ${ }^{3}$ Laboratory for Biomechanics and Tissue Engineering, Department \\ of Biomedical Engineering, Eindhoven University of Technology, P.O. Box 513, Building W-hoog 4.137, 5600 MB Eindhoven, \\ The Netherlands
}

(Received 23 November 2007; accepted 10 December 2008; published online 18 December 2008)

\begin{abstract}
In heart valve tissue engineering, most bioreactors try to mimic physiological flow and operate with a preset transvalvular pressure applied to the tissue. The induced deformations are unknown and can vary during culturing as a consequence of changing mechanical properties of the engineered construct. Real-time measurement and control of local tissue strains are desired to systematically study the effects of mechanical loading on tissue development and, consequently, to design an optimal conditioning protocol. In this study, a method is presented to assess local tissue strains in heart valve leaflets during culturing. We hypothesize that local tissue strains can be determined from volumetric deformation. Volumetric deformation is defined as the amount of fluid displaced by the deformed heart valve leaflets in a stented configuration, and is measured, noninvasively, using a flow sensor. A numerical model is employed to relate volumetric deformation to local tissue strains in various regions of the leaflets (e.g. belly and commissures). The flow-based deformation measurement method was validated and its functionality was demonstrated in a tissue engineering experiment. Tri-leaflet, stented heart valves were cultured in vitro and during mechanical conditioning, realistic values for volumetric and local deformation were obtained.
\end{abstract}

Keywords-Bioreactor, Tissue strain, Flow measurement, Conditioning protocol.

\section{INTRODUCTION}

Contemporary tissue engineered heart valves seem to have sufficient mechanical strength for implantation. However, mechanical properties, tissue structure and architecture still need to be improved. ${ }^{27}$ Research

Address correspondence to J. Kortsmit, Laboratory for Biomechanics and Tissue Engineering, Department of Biomedical Engineering, Eindhoven University of Technology, P.O. Box 513, Building W-hoog 4.105, 5600 MB Eindhoven, The Netherlands. Electronic mail: j.kortsmit@tue.nl has demonstrated that dynamic load application to the developing tissue in a bioreactor system enhances the growth, remodeling and mechanical properties of tissue engineered structures. $3,11,16,18,27$

In heart valve tissue engineering, many bioreactors have been developed and almost all systems try to mimic physiological flow. These systems either apply mechanical loading in a wide physiological range, mimicking both systole as well as diastole, ${ }^{9,15,32}$ or are characterized by simulation of the systolic or opening phase of the cardiac cycle. ${ }^{17,31,35,42}$ However, the diastolic phase or closing phase represents the load bearing phase of the cardiac cycle, in which most strain is applied to the heart valve tissue. The positive effect of cyclic tissue straining on the developing construct has been demonstrated in recent studies. It enhances cell proliferation and functional tissue formation. Furthermore, an increase in ultimate tensile strength and tissue stiffness is observed, compared to static control. ${ }^{21,24,28,29,38,39}$

In a recently developed bioreactor concept, the Diastolic Pulse Duplicator (DPD), ${ }^{25}$ dynamic strains were induced in the heart valve leaflets by applying a dynamic pressure difference over the closed valve. The strain-based conditioning approach was tested by culturing human heart valve leaflets in vitro. The results showed more tissue formation and non-linear tissuelike mechanical properties in the strained valves when compared to unloaded valves. The strain-based conditioning approach of the DPD, in combination with its simplicity in use, offers new possibilities for bioreactor design and optimization of tissue properties in heart valve tissue engineering.

The main shortcoming of current bioreactor systems including the DPD is the lack of control during load application. Preset transvalvular pressures are applied 


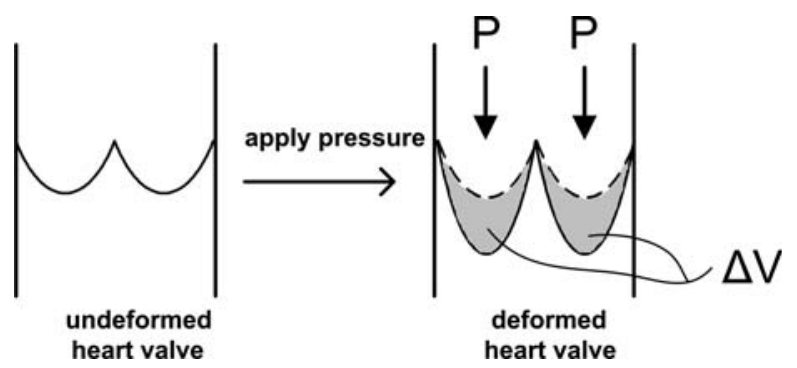

FIGURE 1. Volume change of the heart valve leaflets as a measure for heart valve deformation.

to the developing heart valve while the induced deformations are unknown. Maximum strain values may vary during conditioning as a consequence of changing mechanical properties of the engineered construct. The effects of variation in applied deformation on tissue remodeling are yet unknown. Therefore, real-time, non-invasive measurement and control of local tissue strains is desired. Consequently, the objective of this study is to assess heart valve leaflet deformation, in real-time and non-invasively, during straining under diastolic loading conditions.

In this study, a method is presented to assess local tissue strains in heart valve leaflets. We hypothesize that local tissue strains can be determined from volume changes of the heart valve as a result of load application (Fig. 1). Volume changes are assessed non-invasively, using flow measurements, and represent the volumetric deformation of the leaflets in a stented configuration.

A numerical model ${ }^{7,25}$ is employed to relate volumetric deformation to local tissue strains in the heart valve leaflet. The measurement method was validated and a tissue engineering experiment was performed to demonstrate the feasibility of the measurement method in vitro.

\section{MATERIALS AND METHODS}

\section{Numerical Model}

To test our hypothesis, a quasi-static numerical model of the heart valve leaflets ${ }^{7,25}$ was applied to investigate the relation between volumetric deformation and local tissue strains in the leaflets. Furthermore, to study the influence of the model's input material parameters on this relation, a sensitivity analysis was performed.

\section{Constitutive Law}

In the model, it is assumed that the leaflets are incompressible and therefore the total Cauchy stress $(\boldsymbol{\sigma})$ is split into a hydrostatic pressure $(p)$ and an extra stress $(\tau)$ :

$$
\boldsymbol{\sigma}=-p \mathbf{I}+\tau
$$

To model non-linear mechanical behavior, a nonlinear Neo-Hookean model ${ }^{7,25}$ is used:

$$
\tau=G(\mathbf{B})(\mathbf{B}-\mathbf{I}),
$$

with the shear modulus $G$ calculated from

$$
G(\mathbf{B})=G_{0}\left(\mathbf{I}_{1}(\mathbf{B}) / 3\right)^{n}
$$

with $G_{0}$ and $n$ material parameters. $\mathrm{I}_{1}(\mathbf{B})=\operatorname{trace}(\mathbf{B})$ represents the first invariant of the left CauchyGreen deformation tensor, which is calculated from $\mathbf{B}=\mathbf{F} \cdot \mathbf{F}^{\mathrm{T}}$, with $\mathbf{F}$ the deformation gradient tensor. The parameter $n$ represents the degree of non-linearity of the constitutive equation: $n>0$ indicates stiffening of the material with increasing strains, whereas $n<0$ indicates softening. Note that the classical NeoHookean model is obtained for $n=0$, with $G=G_{0}$.

\section{Balance Equations}

After the material parameters have been determined, finite element analyses (FEA) are performed to simulate the mechanical response of the engineered leaflets. The balance equations that have to be solved are conservation of momentum and mass for an incompressible solid:

$$
\begin{aligned}
\vec{\nabla} \cdot \sigma & =\overrightarrow{0} \\
J-1 & =0
\end{aligned}
$$

where $J=\operatorname{det}(\mathbf{F})$ represents the volume change between the undeformed, stress-free configuration and the deformed configuration. The finite element formulation $^{1}$ was used to solve the set of balance equations. The numerical framework and the constitutive equations were implemented in the software package SEPRAN. ${ }^{37}$

\section{Geometry and Boundary Conditions}

The finite element mesh of the leaflets in the closed configuration consists of only one half of a leaflet, because of symmetry (Fig. 2a). At the symmetry edge, nodal displacements in the normal direction are suppressed (Fig. 2b). At the bottom (ventricular) side of the fixed edge, nodal displacements are suppressed in all directions. At the free edge, a contact surface is defined to model coaptation of adjacent leaflets. The radius of the leaflets is set to $12 \mathrm{~mm}$. The belly and commissural regions of the heart valve are indicated by the selected areas 1 and 2, respectively. To model the diastolic transvalvular load, pressure is applied to the top surface of the leaflets. Subsequently, volumetric and local deformations are calculated. 
(a)

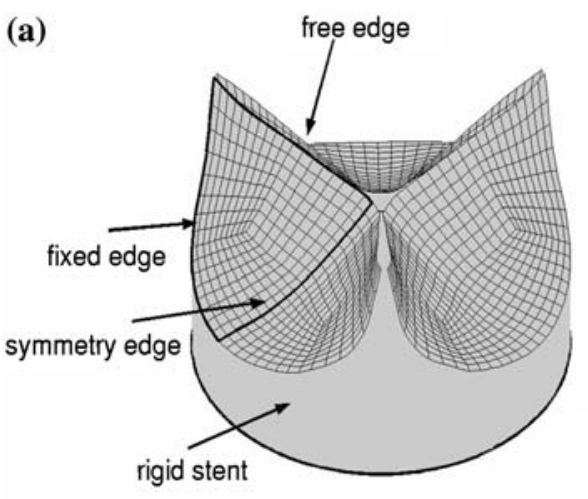

(b)

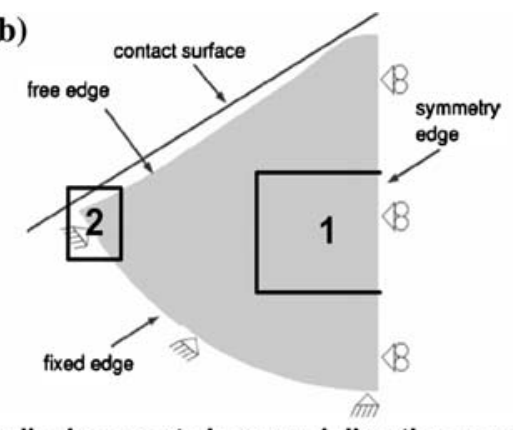

$=$ displacements in normal direction suppressed

蒚 = displacements in all directions suppressed

FIGURE 2. (a) Finite element mesh of the stented heart valve geometry in the closed configuration. In the computations only one half of a leaflet is used because of symmetry. (b) Schematic representation of one half of a heart valve leaflet, showing the loading and boundary conditions. The gray area represents the top (aortic) side of the leaflet to which pressure is applied. A contact surface is defined to model coaptation of the leaflets. ${ }^{7,25}$ Areas 1 and 2 represent the belly and commissural region of the heart valve leaflet, respectively.

TABLE 1. Experimental data and input parameters for the numerical model; thickness $(t)$, shear modulus $\left(G_{0}\right)$ and degree of non-linear material behavior $(n)$ of the heart valve leaflets.

\begin{tabular}{lccc}
\hline & $t(\mathrm{~mm})$ & $G_{0}(\mathrm{MPa})$ & $n$ \\
\hline Experimental data $^{7,8}$ & 0.50 & 0.50 & 10 \\
Numerical model & $0.35-1.0$ & $0.1-100$ & $1-15$ \\
\hline
\end{tabular}

\section{Simulations}

To relate volumetric to local deformations in the heart valve leaflets, simulations were performed. Increasing pressure differences, from 0 to $13 \mathrm{kPa}$, were applied and both volumetric deformation and local tissue strains were computed. To assess regional variations, mean values of maximum principal strain were calculated for the entire valve leaflet, the belly region and the commissural region.

The magnitude of the input parameters, thickness $(t)$, shear modulus $\left(G_{0}\right)$ and degree of non-linearity $(n)$ of the heart valve leaflets were chosen based on experimental data of tissue engineered human valves after 2-4 weeks of culturing (Table 1). ${ }^{7,8}$ To investigate the influence of the model's input parameters on the relation between volumetric and local deformation, a parametric study was performed. The parameters were varied in a range to cover the experimental data (Table 1). The thickness, shear modulus and degree of non-linearity were varied between 0.35 and $1 \mathrm{~mm}$, 0.1 and $100 \mathrm{MPa}$, and between 1 and 15 , respectively. The influence of each parameter was studied by varying it over the given range while the other two parameters were kept constant at $0.5 \mathrm{~mm}, 0.5 \mathrm{MPa}$ and 10 for thickness, shear modulus and degree of nonlinearity, respectively. The calculated volumetric-local deformation curves were averaged and error bars indicated the standard deviation due to the range in input parameters.

\section{Experimental Set-Up}

\section{Bioreactor}

The deformation measurement method was implemented in a bioreactor system similar to the set-up of the Diastolic Pulse Duplicator designed by Mol et al. ${ }^{25}$ Briefly, the bioreactor system consisted of four main components: a proportional compressed-air valve (A), a pulsatile pump encompassing a flexible silicone rubber tube (B), a bioreactor, consisting of two chambers separated by the cultured valve $(\mathrm{C})$ and a medium container (D) (Figs. 3a and 3b). The whole system was filled with $150 \mathrm{~mL}$ of culture medium. The silicone rubber tube, which functioned as a pulsatile pump was compressed and decompressed by dynamic air pressure from the proportional valve. The amount, frequency and waveform of air release were controlled via a programmable multi-IO-card using LABVIEW software (National Instruments, USA). As a result, fluid was taken from the container and injected in the upper chamber of the bioreactor, which caused a dynamic pressure difference over the heart valve. Consecutively, medium exited the bioreactor from both the lower and the upper chamber. Pressure sensors (P10EZ, BD, USA) were positioned in both chambers, while two flow sensors (Transonic Systems Inc., USA) were attached to the tubing through which fluid entered $\left(F_{\mathrm{i}}\right)$ and exited $\left(F_{\mathrm{o}}\right)$ the bioreactor (Figs. 3a and $3 \mathrm{~b}$ ).

\section{Deformation Measurement Method}

Flow measurements were performed to assess volumetric deformation of the heart valve leaflets in a 
(a)

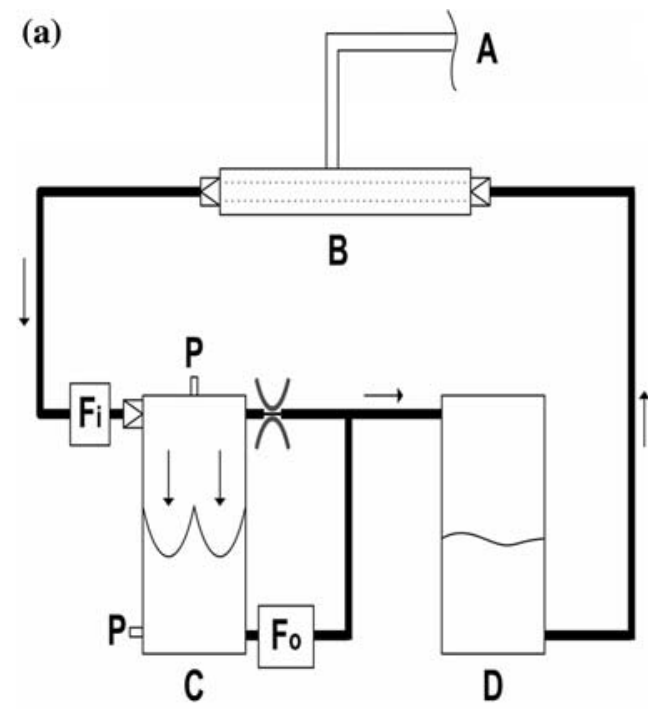

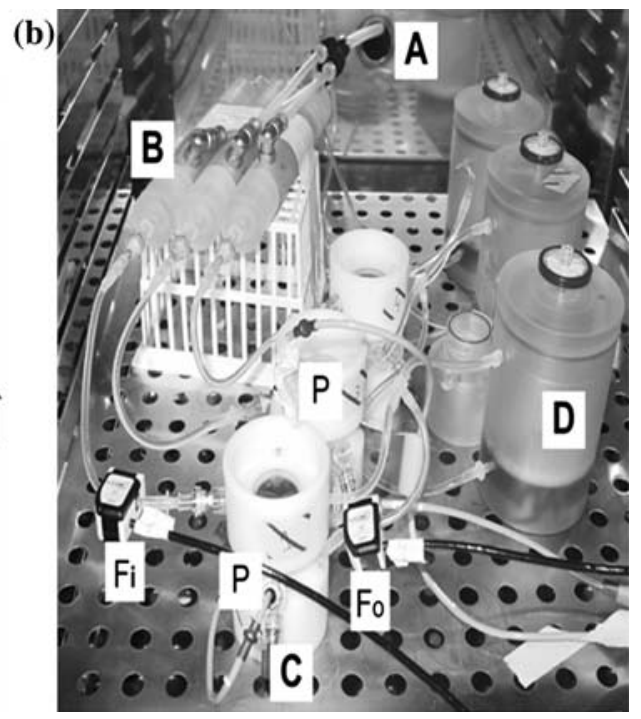

FIGURE 3. (a) Schematic drawing of the bioreactor system and (b) a photograph of 3 systems in use simultaneously. One bioreactor system consists of a compressed air supply (A), a pneumatic pump (B), a bioreactor (C), including 2 pressure sensors $(P)$ and 2 flow sensosr $\left(F_{i}, F_{o}\right)$, and a medium container (D).

(a)

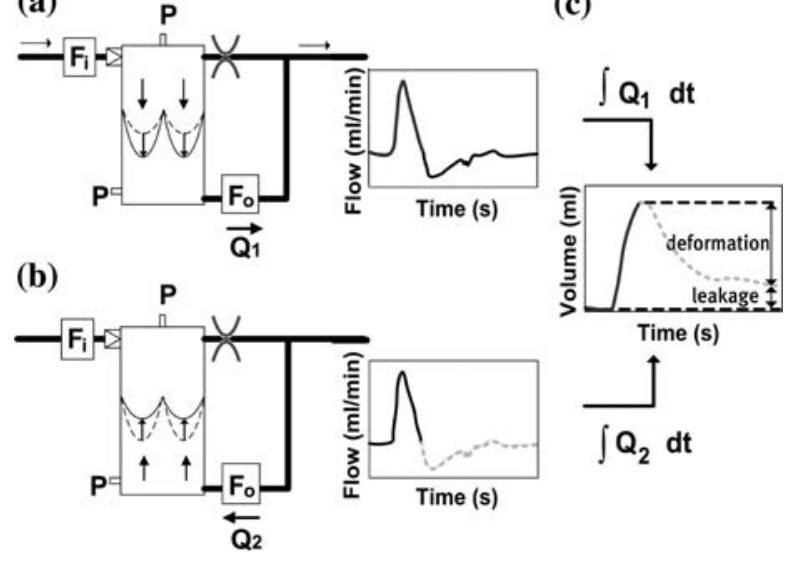

FIGURE 4. Flow-based deformation measurement principle. (a) Medium enters the bioreactor. A pressure difference over the heart valve is created which causes the valve leaflets to bulge down. As a result, a medium flow exits the lower part of the bioreactor $\left(Q_{1}\right)$. (b) After deformation, the heart valve returns to its undeformed state and consequently medium is reentering the bioreactor $\left(Q_{2}\right)$. (c) By time-integration of the measured flow signal, volumetric deformation of the heart valve leaflets can be distinguished from the leakage through the valve.

stented configuration. During load application, a pulsatile flow $\left(F_{\mathrm{i}}\right)$ was injected into the upper chamber of the bioreactor. In combination with the relatively large resistance of the upper chamber exit, this flow caused a dynamic pressure difference over the cultured heart valve. As a result, the heart valve leaflets deformed, and fluid in the lower chamber of the bioreactor was displaced and exited the bioreactor (Fig. 4a). After loading, the heart valve returned to its undeformed state and the displaced fluid reentered the lower chamber of the bioreactor (Fig. 4b). The volumetric deformation of the heart valve was defined as the amount of fluid exiting and subsequently reentering the bioreactor. This deformation was obtained after time-integration of the lower chamber exit flow $\left(F_{\mathrm{o}}\right)$. The net flow leaving the lower chamber was defined as the amount of fluid leaking through the valve. Furthermore, relative leakage was defined as the leak flow through the valve divided by the amount of fluid entering the bioreactor. By using this technique, volumetric deformation was distinguished from leakage (Fig. 4c).

\section{Validation}

To verify the functioning of the deformation measurement method, a validation was performed in two steps. First, the experimentally measured deformation values of a non-leaking rubber heart valve were compared to values calculated with the numerical model. This was done to evaluate the applicability of the measurement technique to assess deformation of a heart valve geometry. Secondly, the influence of leakage on the measurement method was studied by simulation of different leak flows.

\section{Polyurethane Heart Valve}

To validate the deformation measurement method for a heart valve geometry, heart valve leaflets were made of polyurethane and bonded to a rigid polycarbonate stent (Fig. 5). Leakage of the heart valve was prevented by gluing the coaptation areas of the leaflets together.

The valve was placed inside the bioreactor. Pressure differences increasing from 0 to $13 \mathrm{kPa}$ were applied 


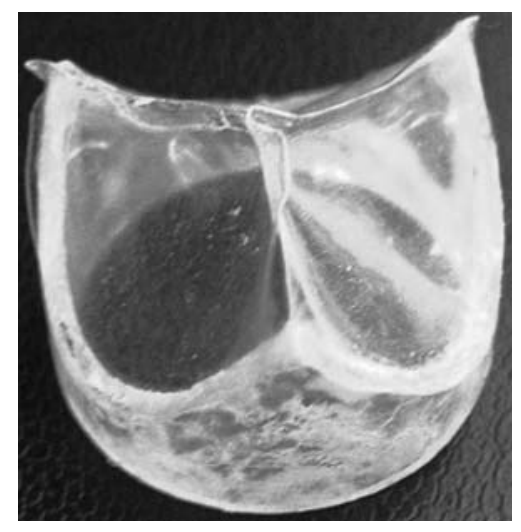

FIGURE 5. Polyurethane heart valve leaflets fixed to a rigid polycarbonate stent.

and the volumetric deformation was determined. The classical Neo-Hookean model (degree of non-linearity; $n=0$ ) was applied to describe the mechanical behavior of the polyurethane valve. The mechanical properties, defined as the product of thickness and shear modulus, of the rubber leaflets was chosen in the range of tissue engineered human valve leaflets. ${ }^{7,8}$ Their thickness was measured $(t=0.08 \pm 0.005 \mathrm{~mm})$ and the shear modulus was determined by performing uniaxial tensile tests $\left(G_{0}=7.2 \pm 0.2 \mathrm{MPa}\right)$. These parameters and the heart valve geometry were used as input for the numerical model and a simulation was performed. The relation between pressure difference and volumetric deformation found in the experiment and the simulation was compared and correlated by the coefficient of determination, $R^{2}$.

\section{Leakage of Polyurethane Disc}

The influence of leakage on the flow-based deformation measurement technique was studied by loading a polyurethane membrane in the bioreactor system. The polyurethane membrane (diameter $=23 \mathrm{~mm}$, thickness $=0.27 \mathrm{~mm}$, DESMOPAN, Bayer AG, Germany) was clamped between two polycarbonate cylinders (height $=21 \mathrm{~mm}$, diameter $=25 \mathrm{~mm}$ ) and inserted into the bioreactor. Increasing pressure differences were applied and deformation was determined by both flow measurement and using an optical technique based on laser Doppler. The laser Doppler velocimeter (Vibrometer OFV 3001, Polytec Optronics Inc., USA) was positioned above the bioreactor. The displacement of the center was measured and the deformation of the membrane was assumed to be semispherical. Therefore, volumetric deformation was computed with:

$$
V=1 / 6 * \pi * h\left(3 r_{1}^{2}+h^{2}\right)
$$

the volume $(V)$ of a segment of a sphere, with $r_{1}$ its radius and $\mathrm{h}$ its height.
Leakage was simulated by connecting the upper and lower chamber of the bioreactor with a bypass. The degree of leakage was controlled by clamping the bypass. Results obtained from both deformation measurement techniques were evaluated for various simulated leaks and applied pressure differences ranging from 0 to $13 \mathrm{kPa}$.

\section{Tissue Engineering Experiment}

To demonstrate the use of the deformation measurement technique in a tissue engineering experiment, stented heart valves with different initial mechanical properties were cultured. Anatomically shaped leaflets were cut out of non-woven polyglycolic acid (PGA; thickness $1.0 \mathrm{~mm}$; specific gravity $70 \mathrm{mg} / \mathrm{cm}^{3}$; Concordia Manufacturing Inc., USA) meshes (thickness: $1.0 \mathrm{~mm}$ ). The dimensions were based on anatomical values measured in human specimens ${ }^{5,34,41}$ and were similar to the computational domain applied in the numerical model. The leaflets were coated with a thin layer of poly-4-hydroxybutyrate (P4HB; molecular weight: $1 \times 10^{6}$ Dalton; TEPHA Inc., USA) and molded in the shape of a trileaflet heart valve. Subsequently, they were attached to a rigid polycarbonate cylinder by sugar leaching of polycaprolactone (PCL; molecular weight: $8 \times 10^{4}$ Dalton; Sigma-Aldrich, USA).

Cells harvested from the human vena saphena magna were expanded using regular cell culture methods ${ }^{36}$ and seeded onto the scaffold using fibrin as a cell carrier. ${ }^{26}$ The medium to culture these cells consisted of DMEM Advanced (Gibco, USA), supplemented with $10 \%$ Fetal Bovine Serum (FBS; PAN Biotech, Germany), 1\% GlutaMax (Gibco, USA), and $0.1 \%$ gentamycin (PAN Biotech, Germany). The medium used for seeding and subsequent tissue culture contained $0.3 \%$ gentamycin and additional L-ascorbic acid 2-phosphate $(0.25 \mathrm{mg} / \mathrm{mL}$; Sigma-Aldrich, USA $)$ to promote extracellular matrix production. ${ }^{25}$ Before seeding, the scaffolds were disinfected in $70 \%$ ethanol and, subsequently, placed in culture medium for 24 or $72 \mathrm{~h}$. Scaffold degradation was promoted by exposure to medium and in this way two heart valves with different initial properties were created.

The constructs were placed in the bioreactor system and subjected to culture medium circulation at low speed $(4 \mathrm{~mL} / \mathrm{min})$ for 5 days. Thereafter, a dynamic pressure difference with constant amplitude (at $1 \mathrm{~Hz}$ ) was applied to the heart valve leaflets for 6 days. After 3 days of mechanical conditioning, the applied load was increased. The pressure difference over the valves, the volumetric deformation of the leaflets and the leak flow through the valves were measured daily. The measured volumetric deformation values were related 
to local tissue strains in the heart valve leaflets using the numerical model.

\section{RESULTS}

\section{Numerical Model}

The relations between volumetric deformation and mean local tissue strain show an increase in the local strain value as a result of an increase in volumetric deformation, in the belly region, commissural region and the entire leaflet (Figs. 6a-6c). The strain values in the commissural region were considerably higher, up to a maximum strain of $22 \%$, compared to the strain values in the entire leaflet $(\max 11 \%)$ and belly region (max 10\%).

Variation of leaflet thickness led to a larger standard deviation in the calculated volumetric deformation-strain curve in all three locations (gray error bars), compared to variation of the other two input parameters. This standard deviation seemed to increase as volumetric deformation increased. In addition, a relatively large standard deviation was also observed in the commissures when the degree of nonlinearity was varied between 1 and 15 (black error bars). The standard deviation resulting from the variation in shear moduli was minimal and, therefore, not shown in Fig. 6.

\section{Validation}

\section{Polyurethane Heart Valve}

The experimentally and numerically obtained relations between volumetric deformation and applied pressure difference over the polyurethane heart valve show that an increase in applied pressure difference led to an increase in volumetric deformation (Fig. 7). A relatively good similarity was seen between the curves $\left(R^{2}=0.977\right)$.

\section{Leakage of Polyurethane Disc}

The volumetric deformation of a disc shaped polyurethane membrane assessed by the flow measurement method is represented as a function of the volumetric deformation measured by laser Doppler velocimetry in Fig. 8. Results are shown for increasing simulated relative leak flows ranging from $L_{0}$ (no leakage) to $L_{7}(53 \%$ leakage). A straight line through the origin with a slope equal to 1 would indicate a perfect resemblance between both measurement techniques and therefore an accurate validation of the flow-based deformation measurement. However, the degree of leakage simulated, seemed to

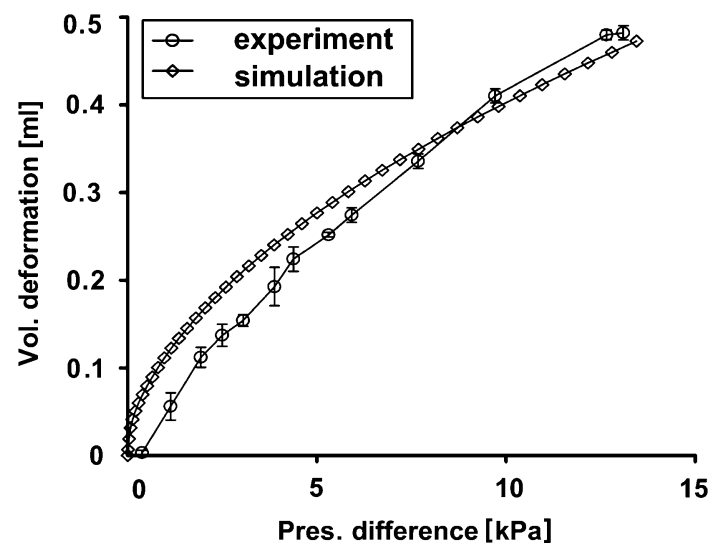

FIGURE 7. Simulated and experimentally obtained relation between volumetric deformation $(\mathrm{mL})$ and applied pressure difference $(\mathrm{kPa})$ over the heart valve. Experimental data $(n=3)$ are depicted by $\bigcirc$ symbols and the standard deviation is represented by error bars. Numerical data are indicated by $\diamond$ symbols. (a)

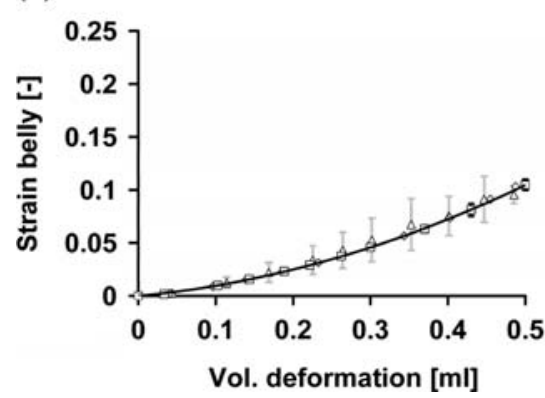

(b)

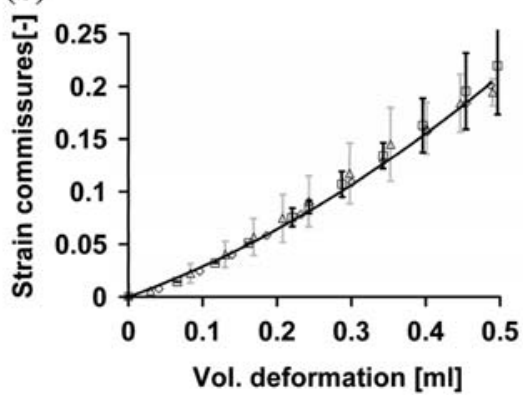

(c)

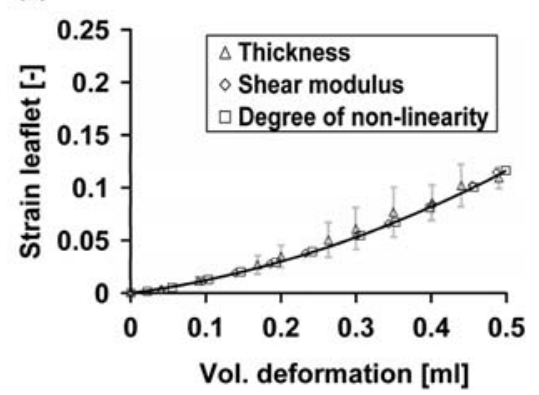

FIGURE 6. Mean maximum principal strain depicted as a function of volumetric deformation (mL). Graphs are obtained by computation of strain and volumetric deformation values when one of the input parameters of the numerical model is varied; thickness $(t)$, shear modulus $\left(G_{0}\right)$ or degree of non-linearity $(n)$ in the range of $t=0.35-1 \mathrm{~mm}, G_{0}=0.1-100 \mathrm{MPa}$ and $n=1-15$, while the other two parameters are kept constant at $0.5 \mathrm{~mm}, 0.5 \mathrm{MPa}$ and 10 , respectively. These graphs are averaged and error bars are added to indicate the standard deviation of the curves due to a variation in thickness (gray error bars) and non-linear material behavior (black error bars). Results are shown for three regions of the valve; belly region (a), commissural region (b) and entire leaflet (c). 
affect the deformation measurements. As leakage increased, a slowly increasing offset in volumetric deformation was observed, which was indicated by a decrease of the coefficient of determination $R^{2}$ (Fig. 8). Furthermore, an increase of the leak flow resulted in a decrease of the imposed maximum pressure difference over the membrane and hence in a downturn of the measured volumetric deformation values.

\section{Tissue Engineering Experiment}

The pressure differences over the cultured heart valves and the induced volumetric deformation values are represented as a function of culture time in Figs. 9a and $9 \mathrm{~b}$, respectively. The results show that for valve 1 , a larger pressure difference led to a smaller deformation compared to valve 2 . This indicated a larger stiffness of valve 1 in comparison with valve 2 . Furthermore, the increase in load application on day 3 led to an increase in volumetric deformation values.

In Fig. 10, the calculated relative leakage through the valve is given as a function of culture time. The graph shows that the relative leakage did not exceed $15 \%$ and was most of the time even less than $10 \%$ for both valves.
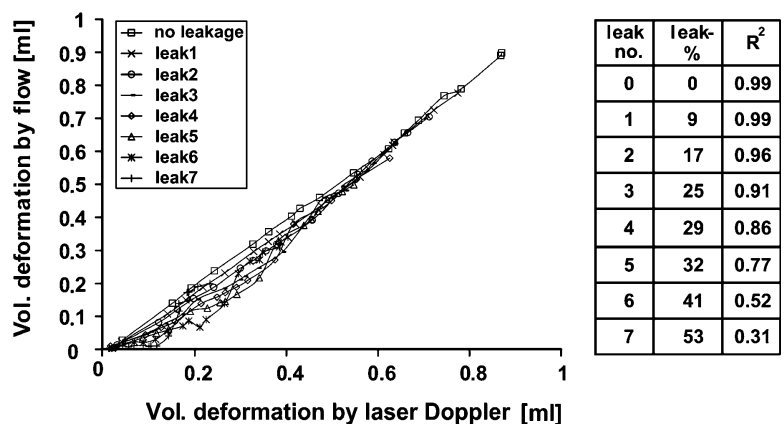

FIGURE 8. Volumetric deformation $(\mathrm{mL})$ of a polyurethane, disc-shaped membrane assessed by the flow-based measurement method is represented as a function of volumetric deformation $(\mathrm{mL})$ measured by laser Doppler. Deformation is determined at different simulated leak flows (leak 0-7) and the resemblance between both measurement techniques at these leak flows is indicated by $R^{2}$. (a)

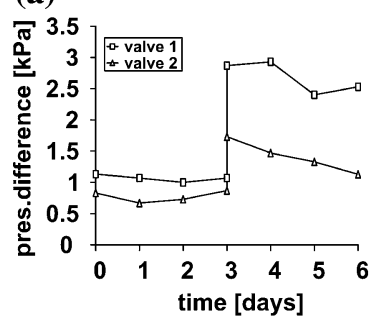

(b)

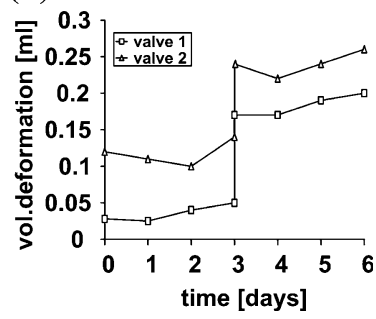

FIGURE 9. (a) Pressure differences (kPa) and (b) volumetric deformation values $(\mathrm{mL})$ represented as a function of dynamic culture time (days) for tissue engineered valves 1 and 2.
The mean local strain values in the entire leaflet, belly and commissures of valves 1 and 2, were determined by the numerical model and are given as a function of culture time (Fig. 11). Comparing the three locations in the leaflet, strains in the commissural region appeared to be larger than in the belly and in the entire leaflet $\left(\varepsilon_{\text {commissures }}>\varepsilon_{\text {leaflet }}>\varepsilon_{\text {belly }}\right)$. In addition for all three locations in the leaflet, valve 2 experienced larger strains compared to valve 1 , which also pointed to the larger stiffness of valve 1 .

\section{DISCUSSION}

In this study, a newly developed measurement method is presented by which leaflet deformation is assessed in heart valve tissue engineering. Deformation is measured using a flow-based measurement technique. This rather simple but new approach offers the possibility to measure deformation in real-time, noninvasively and non-destructively. No studies are known in which deformation of tissue engineered heart valves was determined in such a way. The research in literature that has been performed includes studies in which relatively simple tissue engineered constructs, 2D geometries ${ }^{10,11,24}$ or blood vessels ${ }^{22,23}$ instead of heart valves were subjected to preset deformational changes. Mechanical stimulation in these bioreactor systems shows strong similarity to the way load is applied by uni- or biaxial stretching devices. Tissue deformation is not measured but applied directly by adjusting mechanical loading. On the other hand, deformation and motion of heart valve leaflets were measured in several studies. Two cameras in combination with ink, graphite or projected markers on the valves surface were used to reconstruct $3 \mathrm{D}$ movement of the leaflet surface and/or to calculate 3D surface strains. ${ }^{4,12-14,19,20,33,40}$ Nevertheless, strain was only calculated when the markers were applied invasively, and the focus in these studies was laid on bioprosthetic valves instead of tissue engineered heart valves.

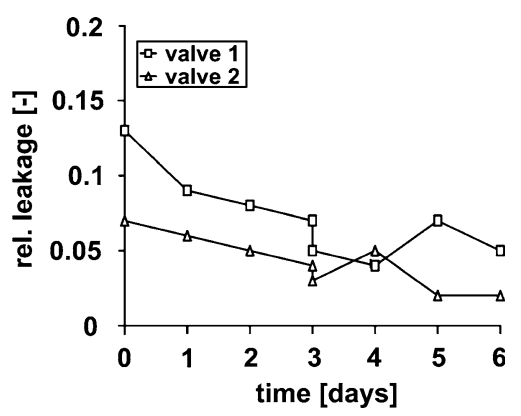

FIGURE 10. Relative leakage, leak flow $(\mathrm{mL})$ of valve divided by inflow $(\mathrm{mL})$ in bioreactor, depicted as a function of culture time (days) for tissue-engineered valves 1 and 2. 
(a)

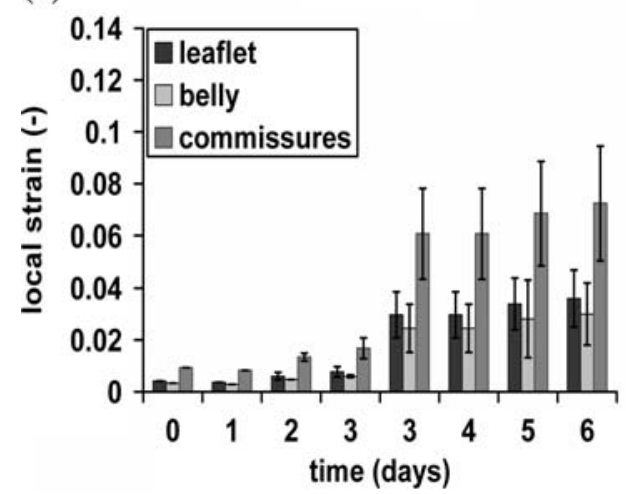

(b)

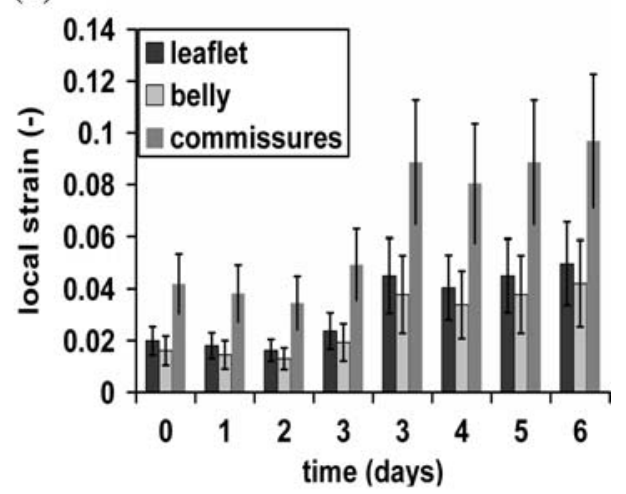

FIGURE 11. Mean local strain values in three leaflet locations, given as a function of culture time (days) for (a) tissue engineered valve 1 and (b) tissue engineered valve 2. Error bars indicate the range (maximum to minimum values) in local tissue strain values as discussed in the 'Numerical Model' section.

A study is known in which deformation of tissue engineered valve leaflets was determined at several conditioning stages. In this study, however, cultured heart valves needed to be sacrificed to estimate the applied strains afterward. ${ }^{8,25}$

Numerical simulations indicated a relation between volumetric deformation and local tissue strains in the heart valve deformation range considered in this study. The relation was relatively insensitive to changes in material properties of the heart valve leaflets. Therefore, local tissue strains in the valve can be directly obtained from measured volumetric deformation values. The conventional numerical-experimental approach $^{30}$ of determining material properties and local strain values by using experimentally measured quantities is very time-consuming and can be avoided by using this direct relation. The relation between volumetric deformation and local tissue strain is most sensitive for variations in thickness; the maximum standard deviation in local strain values at a constant volumetric deformation was $25 \%$. This is probably caused by the relatively large influence of thickness on the flexural behavior of the leaflets when the valve deforms. Furthermore, the largest standard deviation in the strain-volumetric deformation curves was found in the commissural region (maximum standard deviation was $20 \%$ ). This part of the leaflet experiences highest strain values during loading and might therefore be more susceptible to changes in thickness and mechanical behavior.

The flow-based measurement method was validated by assessing deformation of a rubber heart valve. A good agreement was found between the curves in which volumetric deformation was plotted vs. applied pressure differences for numerically and experimentally derived data (Fig. 7). In addition, the numerical model was coupled to the experimental bioreactor set-up and thus local tissue strains were acquired from measured volumetric deformation values. Instead of experimental validation, a numerical model was used to verify the measurement method. Nevertheless, this model has proven its validity and the good match between the results of the experiment and the model is mutually valid.

The results of the second validation experiment, in which the influence of leakage was studied, showed a slightly growing offset in deformation values with increasing simulated leak flows (Fig. 8). This phenomenon is only seen in the volumetric deformation data obtained by the flow-based measurement technique. It is probably caused by the ratio between the amount of fluid exiting the lower bioreactor chamber as a result of leakage, and the amount of fluid reentering the bioreactor when the heart valve leaflets return to their unloaded configuration after load application. As leakage increases this ratio increases and it becomes more difficult, examining the flow data, to distinguish the relatively small volumetric deformation from the large leak flow. Regarding Fig. 8, the flow based deformation measurement method is less accurate for a relative leakage larger than $25 \%$.

Volumetric deformation of engineered heart valve leaflets was related to local tissue strains by using a non-linear Neo-Hookean model to describe the mechanical behavior of the leaflets over time. In this model, the material properties were assumed to be homogeneous and isotropic. The relation between volumetric and local deformation was studied and was applied to tissue engineered heart valves. Preceding research $^{25,27}$ indicates that the material behavior of these valves developed from being homogeneous, isotropic in the first weeks to inhomogeneous, anisotropic behavior after four weeks of culturing. Consequently, assuming homogeneous and isotropic material properties was not correct for the valve leaflets after four weeks of culturing. The use of a structurally based 
anisotropic constitutive model could be a better option to describe material behavior in the final stages of culturing. ${ }^{6}$ When material properties in the circumferential and radial direction of the heart valve are significantly different, the anisotropic model can be applied to assess local deformations by using the conventional numerical-experimental approach, mentioned earlier. ${ }^{30}$

The tissue engineering experiment demonstrated the possibility to measure volumetric deformation of the heart valve leaflets during mechanical conditioning, in real-time and non-invasively. Local strain values assessed by the numerical model were maximal $6 \%$ (belly), 10\% (commissural region) or 7\% (entire leaflet). These values are found to be realistic., ${ }^{2,40}$ Furthermore, relative leakages of the valves were small, generally even less than $10 \%$ (Fig. 10). So, according to Fig. 8, obtained deformation values seem to be accurate. Finally, as a result of various durations of scaffold degradation prior to seeding, two valves with different mechanical properties were cultured. This difference was clearly observed from the pressure and deformation measurement data (Figs. 9 and 11). It indicates that the valve which was exposed to medium longest (valve 2), has the lowest stiffness.

\section{CONCLUSION}

The proposed flow-based deformation measurement method incorporated in the bioreactor system enables us to assess local tissue strains in the leaflets, in realtime, non-invasively and non-destructively, during culturing. By using this technique, the bioreactor system has a great potential to systematically study the effects of mechanical loading on tissue development and, consequently, to design an optimal conditioning protocol for tissue engineering of aortic heart valve replacements.

\section{ACKNOWLEDGMENT}

This research is supported by the Dutch Program for Tissue Engineering.

\section{OPEN ACCESS}

This article is distributed under the terms of the Creative Commons Attribution Noncommercial License which permits any noncommercial use, distribution, and reproduction in any medium, provided the original author(s) and source are credited.

\section{REFERENCES}

${ }^{1}$ Bathe, K. J. Finite Element Procedures. Englewood Cliffs: Prentice Hall, 1996.

${ }^{2}$ Billiar, K. L., and M. S. Sacks. Biaxial mechanical properties of the natural and glutaraldehyde treated aortic valve cusp-part I: experimental results. J. Biomech. Eng.-T. ASME 122(1):23-30, 2000.

${ }^{3}$ Butler, D. L., S. A. Goldstein, and F. Guilak. Functional tissue engineering: the role of biomechanics. J. Biomech. Eng.-T. ASME 122(6):570-5, 2000.

${ }^{4}$ Chen, L., A. D. McCulloch, and K. May-Newman. Nonhomogeneous deformation in the anterior leaflet of the mitral valve. Ann. Biomed. Eng. 32(12):1599-1606, 2004.

${ }^{5}$ Clark, R. E., and E. H. Finke. Scanning and light microscopy of human aortic leaflets in stressed and relaxed states. J. Thorac. Cardiovasc. Surg. 67:792-804, 1974.

${ }^{6}$ Driessen, N. J. B., C. V. C. Bouten, and F. P. T. Baaijens. A structural constitutive model for collagenous cardiovascular tissues incorporating the angular fiber distribution. J. Biomech. Eng.-T. ASME 127:494-503, 2005.

${ }^{7}$ Driessen, N. J. B., A. Mol, C. V. C. Bouten, and F. P. T. Baaijens. Modeling the mechanics of tissue-engineered human heart valve leaflets. In: Proceedings of ICCB 2005, edited by H. Rodrigues, M. Cerrolaza, M. Doblare, J. Ambrosio, and M. Viceconti. Lisbon, Portugal, 2005, pp. $457-476$.

${ }^{8}$ Driessen, N. J. B., A. Mol, C. V. C. Bouten, and F. P. T. Baaijens. Modeling the mechanics of tissue-engineered human heart valve leaflets. J. Biomech. 40(2):325-334, 2007.

${ }^{9}$ Dumont, K., J. Yperman, E. Verbeken, P. Segers, B. Meuris, S. Vandenberghe, W. Flameng, and P. R. Verdonck. Design of a new pulsatile bioreactor for tissue engineered aortic heart valve formation. Artif. Organs 26(8):710-714, 2002.

${ }^{10}$ Engelmayr, G. C., D. K. Hildebrand, F. W. H. Sutherland, J. E. Mayer, and M. S. Sacks. A novel bioreactor for the dynamic flexural stimulation of tissue engineered heart valve biomaterials. Biomaterials 24:2523-2532, 2003.

${ }^{11}$ Engelmayr, G. C., Jr., E. Rabkin, F. W. Sutherland, F. J. Schoen, J. E. Mayer, Jr., and M. S. Sacks. The independent role of cyclic flexure in the early in vitro development of an engineered heart valve tissue. Biomaterials 26(2):175-187, 2005.

${ }^{12}$ Gao, B. Z., S. Pandya, C. Arana, and N. H. C. Hwang. Bioprosthetic heart valve leaflet deformation monitored by double-pulse stereo photogrammetry. Ann. Biomed. Eng. 30:11-18, 2002.

${ }^{13}$ Gao, Z. B., S. Pandya, N. Hosein, M. S. Sacks, and N. H. C. Hwang. Bioprosthetic heart valve leaflet motion monitored by dual camera stereo photogrammetry. J. Biomech. 33:199-207, 2000.

${ }^{14}$ Gao, B. Z., O. Schulz, S. Pandya, and N. H. C. Hwang. A structured light technique for monitoring bioprosthetic heart valve leaflet surface contours. Cardiovasc. Med. 1(1):3-13, 2001.

${ }^{15}$ Hildebrand, D. K., Z. J. Wu, J. E. Mayer, and M. S. Sacks. Design and hydrodynamic evaluation of a novel pulsatile bioreactor for biologically active heart valves. Ann. Biomed. Eng. 32(8):1039-1049, 2004.

${ }^{16}$ Hoerstrup, S. P., R. Sodian, S. Daebritz, J. Wang, E. A. Bacha, D. P. Martin, A.M. Moran, J. Guleresian, J. S. Sperling, S. Kaushal, J. P. Vacanti, F. J. Schoen, and 
J. E. Mayer. Functional living trileaflet heart valves grown in vitro. Circulation 102(suppl III):III-44-III-49, 2000.

${ }^{17}$ Hoerstrup, S. P., R. Sodian, J. S. Sperling, J. P. Vacanti, and J. E. Mayer. New pulsatile bioreactor for in vitro formation of tissue engineered heart valves. Tissue Eng. 6(1):75-79, 2000

${ }^{18}$ Isenberg, B. C., and R. T. Tranquillo. Long-term cyclic distension enhances the mechanical properties of collagenbased media equivalents. Ann. Biomed. Eng. 31:937-949, 2003.

${ }^{19}$ Iyengar, A. K. S., H. Sugimoto, D. B. Smith, and M. S. Sacks. Dynamic in vitro quantification of bioprosthetic heart valve leaflet motion using structured light projection. Ann. Biomed. Eng. 29:963-978, 2001.

${ }^{20}$ Jensen, M. O. J., J. D. Lemmon, V. C. Gessaghi, C. P. Conrad, R. A. Levine, and A. P. Yoganathan. Harvested porcine mitral xenograft fixation: impact on fluid dynamic performance. J. Heart Valve Dis. 10:111-124, 2001.

${ }^{21}$ Kim, B. S., J. Nikolovski, J. Bonadio, and D. J. Mooney. Cyclic mechanical strain regulates the development of engineered smooth muscle tissue. Nat. Biotechnol. 17(10):979-83, 1999.

${ }^{22}$ McCulloch, A. D., A. B. Harris, C. E. Sarraf, M. R. C. Path, and M. Eastwood. New multi-cue bioreactor for tissue engineering of tubular cardiovascular samples under physiological conditions. Tissue Eng. 10(3/4):565-573, 2004.

${ }^{23}$ Mironov, V., V. Kasyanov, K. McAllister, S. Oliver, J. Sistino, and R. Markwald. Perfusion bioreactor for vascular tissue engineering with capacities for longitudinal stretch. J. Craniofac. Surg. 14(3):340-347, 2003.

${ }^{24} \mathrm{Mol}$, A., C. V. C. Bouten, G. Zund, C. Guenter, J. F. Visjager, M. I. Turina, F. P. T. Baaijens, and S. P. Hoerstrup. The relevance of large strains in functional tissue engineering of heart valves. Thorac. Cardiovasc. Surg. 51:78-83, 2003.

${ }^{25} \mathrm{Mol}$, A., N. J. B. Driessen, M. C. M. Rutten, S. P. Hoerstrup, C. V. C. Bouten, and F. P. T. Baaijens. Tissue engineering of human heart valve leaflets: a novel bioreactor for a strain-based conditioning approach. Ann. Biomed. Eng. 33(12):1-11, 2005.

${ }^{26}$ Mol, A., M. I. van Lieshout, G. C. Dam-de Veen, S. Neuenschwander, S. P. Hoerstrup, F. P. T. Baaijens, and C. V. C. Bouten. Fibrin as a cell carrier in cardiovascular tissue engineering applications. Biomaterials 26:3113-3121, 2005.

${ }^{27} \mathrm{Mol}$, A., M. C. M. Rutten, N. J. B. Driessen, C. V. C. Bouten, G. Zund, F. P. T. Baaijens, and S. P. Hoerstrup. Autologous human tissue-engineered heart valves: prospects for systemic application. Circulation 114:152-158, 2006.

${ }^{28}$ Niklason, L. E., W. Abbott, J. Gao, B. Klagges, K. K. Hirschi, K. Ulubayram, N. Conroy, R. Jones, A. Vasanawala, S. Sanzgiri, and R. Langer. Morphologic and mechanical characteristics of engineered bovine arteries. J. Vasc. Surg. 33:628-638, 2001.
${ }^{29}$ Niklason, L. E., J. Gao, W. M. Abbott, K. K. Hirschi, S. Houser, R. Marini, and R. Langer. Functional arteries grown in vitro. Science 284:489-493, 1999.

${ }^{30}$ Oomens, C. W., M. R. van Ratingen, J. D. Janssen, J. J. Kok, and M. A. Hendriks. A numerical-experimental method for a mechanical characterization of biological materials. J. Biomech. 26(4-5):617-621, 1993.

${ }^{31}$ Rabkin, E., S. P. Hoerstrup, M. Aikawa, J. E. Mayer, and F. J. Schoen. Evolution of cell phenotype and extracellular matrix in tissue-engineered heart valves during in vitro maturation and in vivo remodeling. J. Heart Valve Dis. 11:308-314, 2002.

${ }^{32}$ Rutten, M. C. M., M. W. Wijlaars, G. J. Strijkers, G. W. M. Peters, C. V. C. Bouten, and F. P. T. Baaijens. The valve exerciser: a mechanics-based bioreactor for physiological loading of tissue-engineered aortic valves. In: Abstract, 13th conference of the European Society of Biomechanics. Wroclaw, Poland, 2002.

${ }^{33}$ Sacks, M. S., Z. He, L. Baijens, S. Wanant, P. Shah, H. Sugimoto, and A. P. Yoganathan. Surface strains in the anterior leaflet of the functioning mitral valve. Ann. Biomed. Eng. 30:1281-1290, 2002.

${ }^{34}$ Sauren, A. A. H. J. The mechanical behaviour of the aortic valve. Ph.D. thesis, Technische Hogeschool Eindhoven, 1981.

${ }^{35}$ Schenke-Layland, K., F. Opitz, M. Gross, C. Doring, K. J. Halbhuber, F. Schirrmeister, Th. Wahlers, and U. A. Stock. Complete dynamic repopulation of decellularized heart valves by application of defined physical signals - an in vitro study. Cardiovasc. Res. 60:497-509, 2003.

${ }^{36}$ Schnell, A. M., S. P. Hoerstrup, G. Zund, S. Kolb, R. Sodian, J. F. Visjager, J. Grunenfelder, A. Suter, and M. Turina. Optimal cell source for cardiovascular tissue engineering: venous vs. aortic human myofibroblasts. Thorac. Cardiovasc. Surg. 49:221-225, 2001.

${ }^{37}$ Segal, A. SEPRAN User Manual, Standard Problems and Programmers Guide. Leidschendam, The Netherlands: Ingenieursbureau SEPRA, 1984.

${ }^{38}$ Seliktar, D., R. A. Black, R. P. Vito, and R. M. Nerem. Dynamic mechanical conditioning of collagen-gel blood vessel constructs induces remodeling in vitro. Ann. Biomed. Eng. 28(4):351-362, 2000.

${ }^{39}$ Seliktar, D., R. M. Nerem, and Z. S. Galis. Mechanical strain-stimulated remodeling of tissue-engineered blood vessel constructs. Tissue Eng. 9(4):657-666, 2003.

${ }^{40}$ Sun, W., A. Abad, and M. S. Sacks. Simulated bioprosthetic heart valve deformation under quasi-static loading. J. Biomech. Eng.-T. ASME 127(6):905-914, 2005.

${ }^{41}$ Thubrikar, M. The Aortic Valve. Boca Raton: CRC Press, 1990.

${ }^{42}$ Zeltinger, J., L. K. Landeen, H. G. Alexander, I. D. Kidd, and B. Sibanda. Development and characterization of tissue-engineered aortic valves. Tissue Eng. 7:9-21, 2001. 